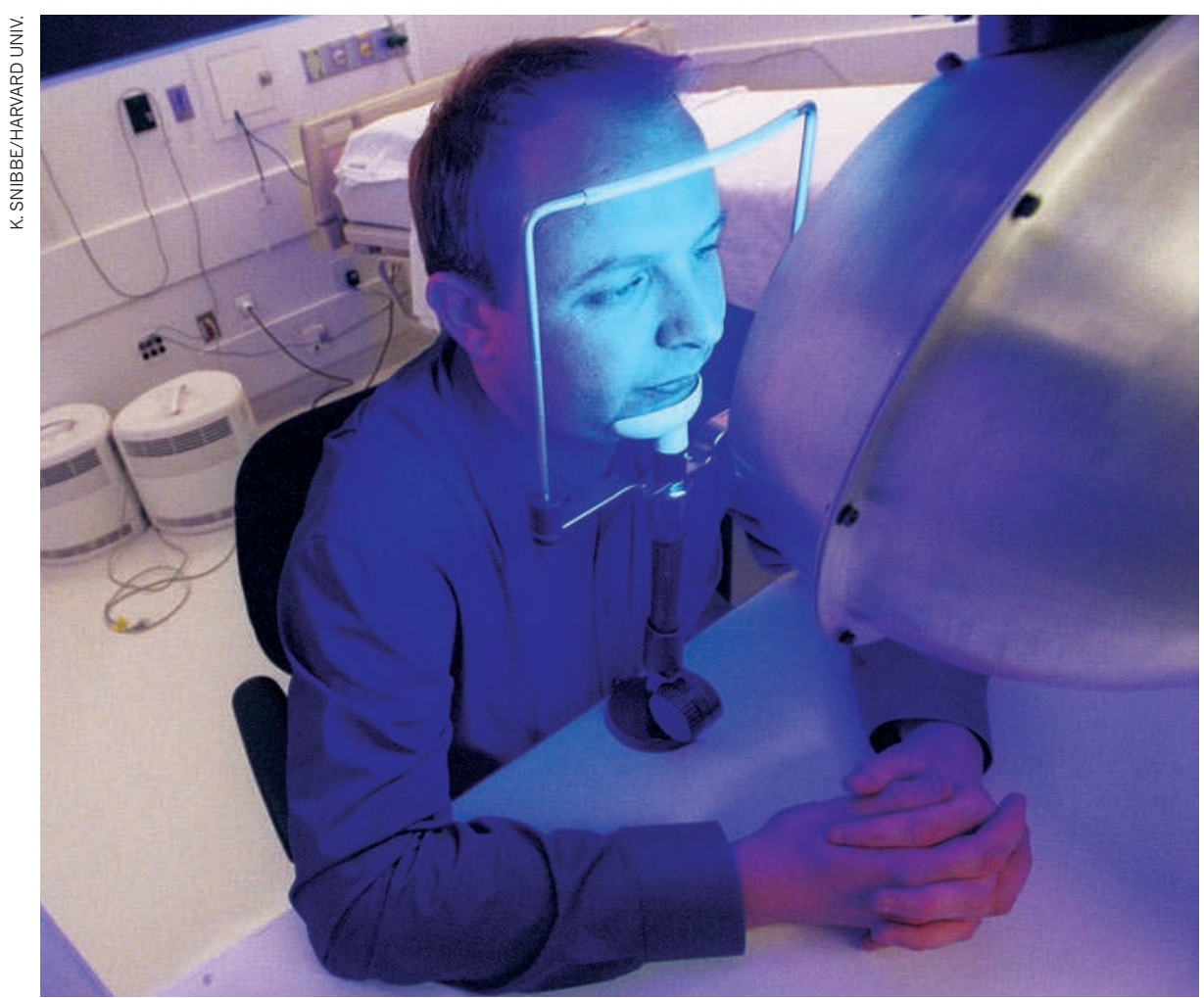

Steven Lockley demonstrates an experimental set-up for studying light-sensitive cells in the eye.

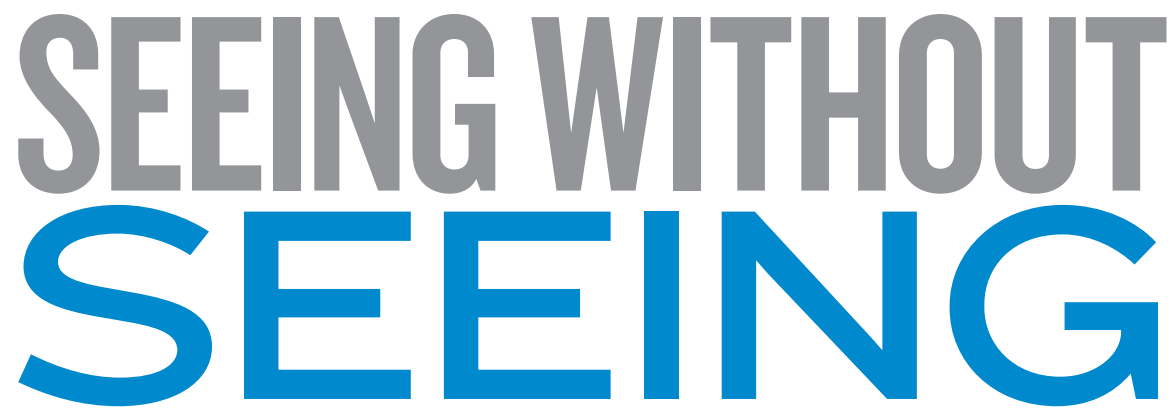

There is more to the eye than rods and cones - the discovery
of a third photoreceptor is rewriting the visual rulebook.

BY CORIE LOK

ussell Foster remembers his first human subject, an 87-year-old woman, as she sat in a dark room facing a backlit pane of frosted glass. A genetic disorder had destroyed the light-sensing rod and cone cells in her eyes, leaving her blind for the past 50 years. She was convinced that she would see nothing. But as the wavelength of light in the room shifted to blue, she reported - after some hesitation - a sort of brightness.

"That just blew us away," says Foster, a neuroscientist at the University of Oxford, UK, and one of the senior authors of a 2007 study reporting the finding ${ }^{1}$.

Foster and his collaborators had done nothing to treat the woman's blindness. Instead, her awareness of light owed itself to a class of light-sensitive cells discovered in 2002. Studies of these intrinsically photosensitive retinal for a third type of photoreceptor. In the early 1990s, while at the University of Virginia in Charlottesville, his lab tested the circadian light responses in a mouse mutant with retinas that degenerate over time, and found that they were indistinguishable from the responses in mice with normal retinas. But light had no effect on the internal clocks of mice whose eyes had been removed ${ }^{2}$.

Scepticism was strong. Foster recalls people walking out during a talk he gave. Critics of the research argued that the mutant mice probably retained some rods and cones that could be setting the clock. So in 1999, Foster, who had moved to Imperial College London, crossed transgenic mice that had no cone cells with mice that had degenerative rod cells, thus eradicating both cell types in the offspring. As long as the mice had eyes, they still had normal circadian rhythms ${ }^{3,4}$.

The next year, Ignacio Provencio, a former graduate student of Foster's now at the University of Virginia, Charlottesville, identified the light-sensitive molecule melanopsin in the mouse and primate ganglion layer ${ }^{5}-$ a network of retinal cells that was only thought to relay signals from rods and cones to the brain (see 'Light in layers'). The presence of this 'photopigment' suggested that some of these cells might also sense light and serve as a new class of photoreceptor. Researchers raced to isolate the cells and show that they could fire in response to light, without input from rods and cones.

The race ended in a tie in 2002. Samer Hattar, a neuroscientist at Johns Hopkins University in Baltimore, Maryland, and his colleagues found that as many as $1 \%$ of the cells in the mouse ganglion layer express melanopsin, which is most sensitive to blue light ${ }^{6}$. David Berson, a neuroscientist at Brown University in Providence, Rhode Island, and his lab showed that these cells, ipRGCs, detect light on their own and reach into the brain's pacemaker, the suprachiasmatic nucleus ${ }^{7}$. The two papers helped to win over the sceptics, says Russell Van Gelder, a neuroscientist and ophthalmologist at the University of Washington, Seattle. “Things really took off in 2002," he says.

Researchers began to develop mouse models in which they could selectively block input from each of the three photoreceptor types in the eye, to probe their individual contributions. But rather than distributing jobs neatly between cell types, the cells seem to swap roles under different conditions.

It became clear that under low light conditions, rods can set the body's clock, but some groups have suggested that under different conditions cones can as well. Perhaps more surprisingly, researchers have found that ipRGCs may contribute to visual perception. Hattar and others fluorescently labelled ipRGCs in mice to trace the projections of these cells to the brain. They found that ipRGCs reach into more brain regions than expected, including 
centres involved in visual processing: the dorsal lateral geniculate nucleus (LGN) and the superior colliculus. Mice without functioning rods and cones, but with intact ipRGCs, could even discriminate patterns in a visual test ${ }^{8}$.

This is puzzling. Melanopsin responds slowly - on the order of seconds - to changes in light, limiting its ability to signal changes in spatial information, says Robert Lucas, a neurobiologist at the University of Manchester, UK. He and his group found that in mice that lacked the gene for melanopsin, and therefore had non-photoreceptive ipRGCs, almost half of the neurons in the LGN had defective light responses. The mice were unable to track background light levels, especially in the daylight range, suggesting that ipRGCs could be encoding information about brightness ${ }^{9}$.

Researchers now think that ipRGCs and rods compensate for each other and may collectively be allowing the eyes and brain to respond to light across a wide range of brightness levels. Why these different photoreceptors share the load in such specific ways is not clear. For example, the sensitivity of ipRGCs to blue light may make them better suited to detect the arrival of dawn and dusk.

\section{BEGINNING TO SEE THE LIGHT}

The ipRGCs might influence phenomena beyond vision and circadian rhythms. Many physiological responses have been linked to light, such as sleep, migraine pain and seasonal affective disorder, and these have recently been associated with ipRGC activity. "There's likely to be a whole array of physiology that, to some degree, is light sensitive," says Provencio.

Learning and memory may be improved under certain light conditions. Provencio and his colleagues presented data last year showing the effects of light on a mouse model for learned fear. Mice were conditioned to associate a mild electric shock with a tone cue. Those that had learned fear in the presence of light froze for longer in response to the tone than those that had been conditioned in the dark. This effect did not appear in mice engineered to lack rods and cones, but did in melanopsinknockout mice, suggesting that the rods and cones are driving this light-enhanced learning. Still, the researchers have not ruled out a role for ipRGCs. These cells route information from the eyes to the non-visual centres in the brain, including those involved in fear responses.

Hattar has unpublished data to suggest that activating melanopsin with light at various points in the sleep-wake cycle of mice impairs learning and memory, even when the animals have normal circadian rhythms. This could mean that exposure to light at times when the body isn't expecting it can be disruptive. And for humans, who have a smaller percentage of ipRGCs than mice, experiments are beginning to show how the cells might contribute to physiology and behaviour. Steven Lockley, a neuroscientist at Brigham

\section{LIGHT IN LAYERS}

Light passes through the ganglion layer and cells in the inner retina to the predominant photoreceptors in the eye - the rods and cones. These then send visual information back to ganglion cells, which transmit it to visual and non-visual centres of the brain. A subset of ganglion cells, called intrinsically photoreceptive retinal ganglion cells (ipRGCs), contain a photopigment, melanopsin, and can also encode and transmit information about light directly.

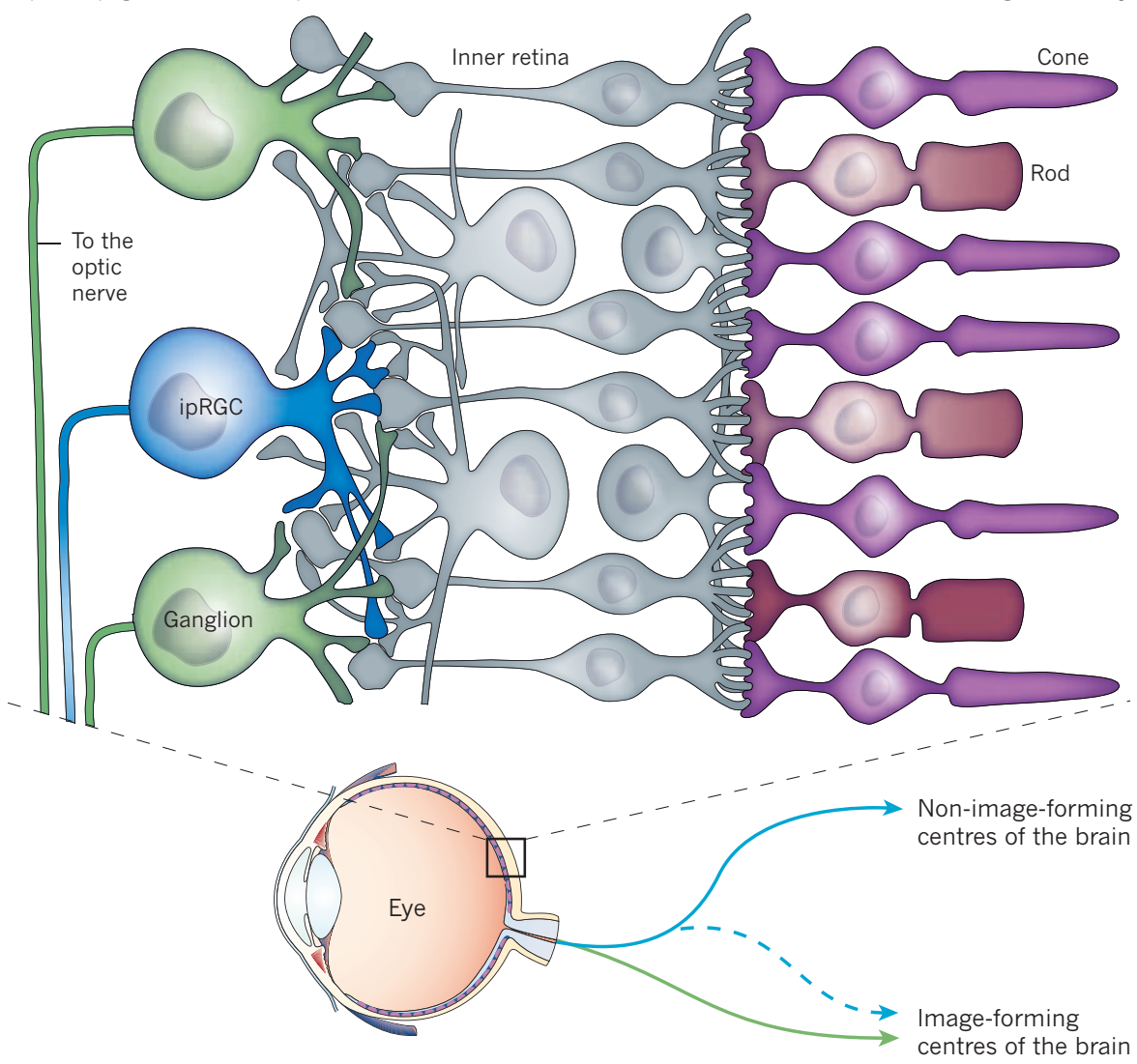

and Women's Hospital in Boston, Massachusetts, and his colleagues tested the reaction times of 16 healthy volunteers while they were exposed to either blue or green light for 6.5 hours. Those exposed to blue light had faster reaction times and fewer attention lapses when they were asked to report when they heard a sound ${ }^{10}$.

Lockley says that these different strands of research might eventually help to engineer 'healthier' light - using specific wavelengths, intensities or even patterns to activate brain pathways and improve mood, sleep or mental performance. "This research opens up a whole new field in terms of light applications, both for use therapeutically and for the general population," says Lockley.

Light of certain frequencies can have beneficial effects, but may also be detrimental to health. Lockley has been working with a group of light engineers, neuroscientists and ophthalmologists, who call themselves the Blue Light Group. They met for the first time this summer to discuss, among other things, any safety issues surrounding blue light, including the idea that excessive exposure to it might contribute to a type of vision loss known as macular degeneration. Many lightemitting diodes, a leading technology for energy-efficient lighting, are rich in blue light, points out Charles Hunt, a materials scientist at the University of California, Davis, who leads the group. Could their wider adoption lead to health problems for people?

Humans have evolved to live under natural light, says Van Gelder. "Could we be doing some damage to our health by poisoning the world with wavelengths that we're not evolved to live in?" he asks. Given the emergence of new kinds of lighting, Hunt says that it is important to find out. "We need answers quickly," he says.

Corie Lok is Nature's Research Highlights editor.

1. Zaidi, F. H. et al. Curr. Biol. 17, 2122-2128 (2007).

2. Foster, R. G. et al. J. Comp. Physiol. A 169, 39-50 (1991).

3. Freedman, M. S. et al. Science $\mathbf{2 8 4 , 5 0 2 - 5 0 4}$ (1999).

4. Lucas, R. J., Freedman, M. S, Muñoz, M., GarciaFernández, J. M. \& Foster R. G. Science 284, 505-507 (1999).

5. Provencio, I. et al. J. Neurosci. 20, 600-605 (2000)

6. Hattar, S., Liao, H.-W., Takao, M., Berson, D. M. \& Yau, K.-W. Science 295, 1065-1070 (2002).

7. Berson, D. M., Dunn, F. A. \& Takao, M. Science 295, 1070-1073 (2002).

8. Ecker, J. L. et al. Neuron 67, 49-60 (2010).

9. Brown, T. M. et al. PLoS Biol. 8, e1000558 (2010)

10.Lockley, S. W. et al. Sleep 29, 161-168 (2006). 